\title{
Effectiveness of Mental Model-Based Training for Complex System Operators: A Quasi-Experimental Study
}

\author{
Xiaomin Lin ${ }^{1,2}$, Xinwen Bai ${ }^{1}$ \\ ${ }^{1}$ Key Laboratory of Behavioral Science, Institute of \\ Psychology, Chinese Academy of Sciences \\ 16 Lincui Road, Chaoyang District, Beijing, P.R. China \\ ${ }^{2}$ University of Chinese Academy of Sciences \\ 19A Yuquan Road, Shi Jingshan District, Beijing, P.R. \\ China \\ ${ }^{1}$ linxm@psych.ac.cn \\ 2 baixw@psych.ac.cn
}

\author{
Jianming $\mathrm{Hao}^{3}$ \\ ${ }^{3}$ Data Center(Beijing), China UnionPay Co. Ltd. \\ Building 22nd, Zark, NO.8 Dongbeiwang West Road, \\ Haidian District, Beijing, P.R. China \\ 3 jmhao@unionpay.com
}

\begin{abstract}
Previous research has indicated that operators of complex systems will achieve higher system operation efficiency and better job performance when they hold high quality mental models. Authors of recent studies have called for greater attention toward how to improve the development of mental models especially intervention factors influencing mental model quality. The current study aimed to develop a new training strategy called mental model-based training and to examine its effectiveness by conducting a quasi-experimental study. Results demonstrated that operators exposed to mental model-based training showed more frequent reflective and learning behaviors in later work than those receiving no training, whereas mental model-based training did not result in superior mental model quality over the control condition. Findings were discussed in terms of the advantages of the mental model-based training strategy as well as its applications in complex systems.
\end{abstract}

Keywords-mental model, mental model-based training, complex system operator

\section{INTRODUCTION}

Large-scale complex systems have been widely employed in different types of organizations, such as nuclear power, air traffic control, chemical engineering, telecommunications and online transaction. The efficient operation of these complex systems highly depends on a small number of strictly trained operators. They are responsible for monitoring complex systems, performing routine procedures, and quickly responding to non-routine situations. In order to cope with high-workload or emergent situations which derive from more and more frequent human-machine interaction, the operators have to develop mental models (hereafter referred to as MMs) of their own to make proper judgment and decision-making [1]. Mental model has been defined as "mechanisms whereby humans are able to generate descriptions of system purpose and form, explanations of system functioning and observed system states, and predictions of future system states" [2]. Previous literature have found that higher performing operators have higher quality MMs, which are more similar to the "expert model". Experts generally solve problems more effectively because their well-structured, easily activated knowledge allows for efficient search of a solution.

Whereas empirical research has confirmed that MM of higher quality generally contributes to better job performance [3, 4], an emerging body of research has examined the antecedents of MM quality, most of which investigates the impacts of individual characteristics including working experience [5], competence [6] and contextual factors, such as acute stress [7]. However, little attention has been paid to understand how to enhance MM quality through interventions. Given the importance of high quality MM in complex systems, it is necessary to develop intervention techniques to facilitate MM quality. Some intervention methods were employed in empirical research such as leader briefings [8], experiential training (hands-on learning) [9] and guided team self-correction training [10]. Findings indicated that they contributed to the enhancement of MM quality to some extent. Among various interventions, cross-training in which team member is trained in the duties of his or her teammates to enhance inter-positional knowledge is one of the most effective methods to foster MM development [3, 11].

Although cross-training method was useful as referred to above, potential shortfalls also existed. On the one hand, it is not necessary to train each operator to gain inter-positional knowledge, since complex systems require experts with specialized skills in one field rather than generalists with a wide range of knowledge and skills associated with all duties. On the other hand, it is neither efficient nor possible for operators to spend much time and effort to learn about the details of other teammates' roles and responsibilities. After all, the amount of time and resources is always limited and cannot be spent in developing interchangeable expertise among operators. Moreover, inter-role knowledge builds much redundancy into a team's expertise which would have a negative impact on effective team functioning. In summary, cross-training strategy, which is essentially knowledge-based, is unpractical and infeasible in complex systems situation.

In the current study, we aimed to explore a new training strategy which helped to improve MM quality. This new training method was referred to as "mental model-based training" (hereafter referred to as MM-based training), which 
was built on Smith-Jentsch's (2001) prior work of the computer-based training on team mental model [5]. Specifically, we used operator's own MM as training materials. Through self-reflection and group discussion, operators corrected their own MM gradually, and finally developed expert model of high quality. The newlydeveloped MM-based training strategy has three distinctive characteristics. First, it reorients to focus more on changing one's knowledge structure or ways of thinking rather than teaching specific knowledge or a finite set of skills per se. Second, it raises operators' awareness to see things from others' perspectives, which helps them better interact with teammates in other position within the team. Third, this new training approach provides them with a comprehensive and holistic understanding of system functioning and system states, as well as how sub-systems interrelate with each other, which is critical to prevent errors and improve effectiveness.

In this study, we employed the quasi-experimental design to evaluate the effectiveness of our newly-developed training strategy. We used Kirkpatrick's (1996) model of training evaluation and criteria $[12,13]$ to choose evaluation criteria that could capture multiple facets and different stages of the training effectiveness. Specifically, we chose three types of criteria, i.e., training satisfaction (reaction criterion), reflective and learning behaviors (behavior criterion), and MM quality (cognition criterion). Accordingly, we expected that compared to those in no-training condition, operators receiving MM-based training will have a positive attitude toward the MM-based training (Hypothesis 1), show more reflective (Hypothesis 2a) and learning behaviors (Hypothesis 2b), and develop MMs of higher quality (Hypothesis 3).

\section{METHODS}

\section{A. Participants and Design}

We conducted this study in the data center of a large hitech corporation in China, where a complex system is established to support online transaction and settlement. The whole system consists of five inter-connected sub-systems, consequently all operators are classified into five departments which correspond to the management of each of the five sub-systems. Five operators, one from each of the five departments, form a team to deal with routine system maintenance, status monitoring and emergency management. Twelve-hour shift system is implemented in this company. Four teams are formed to work on a rotating shift for a month, and at the end of each month, these teams are dismissed. These operators will work on the regular nine-tofive shift in the following month. At the same time, four new teams are formed for the rotating shift. A total of 46 operators were invited to participate in this study. All of them were male with mean age of 30.40 years $(S D=4.80)$ and mean tenure of 7.30 years $(S D=5.53)$.

Because of practical constraints, we employed a quasiexperimental design to examine the effect of MM-based training. 23 operators happened to work on rotating shift in the upcoming month were assigned to training condition. The other 23 operators who worked regularly in that month were treated as control group (no-training condition). There were no significant difference on demographic variables such as gender, age, education degree, and tenure between training and control group. Operators in training condition participated a two-stages MM-based training which lasted one hour at each stage. Operators in control group received no such training.

\section{B. Measure}

Mental model quality. Following prior work of MM measurement, we used paired comparison ratings method to measure MM quality [3, 6]. First, we used behavioral event interview technique to separately interview 5 supervisors and 5 front-line operators to pinpoint the critical characteristics of system operation. As a result, we learnt that coping with emergency was the most challenging situation for operators. Once system fails to perform, five subsystem platforms will report corresponding fault phenomena, then five on-shift operators have to make a joint judgment to identify the cause of system fault. The key to deal successfully with emergency lies in deeply understanding the correlation between fault phenomena from different sub-systems. Therefore, system fault phenomena will be used as stimulus materials to elicit and represent individual MM. Specifically, we invited 5 supervisors to elaborately select 10 core fault phenomena from the case library which was used as training materials or routine simulation exercise for operators.

Second, using paired comparison ratings, operators was asked to make judgments about the relatedness of each pair of fault phenomenon statements by using a two points scale in which "0" represented "not related" and " 1 " meant "related", according to their own experience and understanding. Following prior work, these matrix data were submitted to UCINET, a network analysis program, to represent each operator's MM. UCINET can also produce a visualized mental model profile in which nodes represent fault phenomenon statements and links represent relations between fault phenomenon statements. As a result, the MM profile vividly demonstrates operator's understanding of the relatedness among pairs of fault phenomena.

Consistent with the extant literature [6], we defined MM quality as the extent to which MM was similar to expert mental model. Expert MM had been determined previously using subject matter experts who were highly proficient in the system operation and maintenance. In line with previous research, we used QAP correlations index (Quadratic Assignment Procedure correlation) to calculate the similarity between operator's individual MM and expert MM, which ranged from -1 (completely inconsistent) through 0 (no consistency) to +1 (completely consistent), with higher values indicating higher quality.

Reflective and learning behaviors. Reflective and learning behaviors scales were administered in both groups. Operators were asked to report the frequency of their reflective and learning behaviors at work in the last month. Two items were included in reflective behavior scale like "I reflect upon myself when working in an ineffective way" and "I think about how to improve the quality and efficiency of my work". Learning behavior scale was adapted from Team 
Learning Behavior Scale [14], which had three items such as "I do my work considering information provided by my colleagues". Both scales ranged from 0 (never) to 5 (always).

Training satisfaction. After training, operators filled out a training satisfaction questionnaire to access their attitude towards the training program. Training satisfaction was measured with four items designed for this study: "The training meets my expectation"; "The training is helpful to my duties"; "The training inspires me to reflect on the relationship among different subsystems"; "The training raises my awareness of taking others' perspectives to see things". Items were answered on 5-point scales ranging from 1 (strongly disagree) to 5 (strongly agree).

\section{Procedure}

Two weeks before the training, all 46 operators were required to take the pre-test of $\mathrm{MMs}$, i.e., the paired comparison ratings of relatedness among a set of 10 fault phenomena statements. The MM profiles were further used as materials for the MM-based training.

Five operators of the same team together attended the MM-based training just before the team started the new shift for the upcoming month. The training consisted of two stages, one-hour each. At stage 1, operators were instructed to reflect on their own MMs individually. First, they were presented with their own MM profiles. They were encouraged to think about why some fault phenomena became a cluster but others were not. Second, they were presented with a team-aggregated MM profile which was achieved by using members' aggregated paired comparison ratings in pre-test. Then operators were required to clarify the differences between one's MM and the prescriptive team-aggregated MM.

Stage 2 resolved around team reflection and discussion. Specifically, 5 operators were told to discuss the differences between each other. Then the team, as a whole, made paired comparison ratings among the same set of fault phenomena. This matrix data was immediately submitted to UCINET to produce a team MM profile. Furthermore, the team was presented with the expert MM profile, and was required to discuss the differences. Finally the team was required to reach final consensus on the team MM profile. The operators were encouraged to apply what they had learnt through the training to the upcoming duty shift. Then they were dismissed and the training session concluded.

Evaluation criteria of training effectiveness were measured at two times. Training satisfaction was measured immediately after training session concluded. Reflective and learning behaviors were administrated two weeks after the end of month-long duty shift. Post-test of MM quality was administrated at this time using a different set of fault phenomena from pre-test. Operators in control group received no MM-based training, thus did not fill in the training satisfaction questionnaire. However, they did complete pre- and post-test of MM quality, and reflective and learning behavior measures as experimental group did.

\section{RESULTS}

As expected, results showed that operators participated in the training program were quite satisfied with the training (mean score $=4.31$ on a 5 -point scale).

To examine the effect of MM-based training on individual behaviors at work, a series of independentsamples $t$-tests were conducted. In line with hypothesis $2 \mathrm{a}$, there were significant differences in reflective behaviors across two conditions $\left(t_{1}(44)=2.38, p<0.05 ; t_{2}(44)=2.20\right.$, $p<0.05)$. As expected in hypothesis $2 \mathrm{~b}$, operators receiving training were more likely to seek help and learn from colleagues than their counterparts in no training condition $\operatorname{did}\left(t_{1}(44)=2.42, p<0.05 ; t_{2}(44)=1.93, p<0.10 ; t_{3}(44)=\right.$ $1.69, p<0.10)$. Thus, hypothesis $2 \mathrm{a}$ and $2 \mathrm{~b}$ were both supported. Means and standard deviations concerning reflective and learning behaviors across two conditions were reported in Table $\mathrm{I}$.

TABLE I. MEANS, STANDARD DEVIATIONS OF REFLECTIVE AND LEARNING BEHAVIORS IN EXPERIMENTAL GROUP AND CONTROL GROUP

\begin{tabular}{lcc}
\hline & $\begin{array}{c}\text { Experimental } \\
\text { group } \\
M(S D)\end{array}$ & $\begin{array}{c}\text { Control } \\
\text { group } \\
M(S D)\end{array}$ \\
\hline $\begin{array}{l}\text { Reflective behavior } \\
\text { 1. I reflect upon myself when working in } \\
\text { an ineffective way. }\end{array}$ & $3.78(0.74)$ & $3.17(0.98)$ \\
2. I think about how to improve the & $4.17(0.49)$ & $3.74(0.81)$ \\
$\quad$ quality and efficiency of my work. & & \\
$\begin{array}{l}\text { Learning behavior } \\
\text { 1. I do my work considering information } \\
\text { provided by my colleagues. }\end{array}$ & $4.35(0.57)$ & $3.87(0.76)$ \\
2. I consider feedback from my \\
$\quad \begin{array}{l}\text { colleagues. } \\
\text { 3. I ask for help from my colleagues } \\
\text { when something comes up that I don't } \\
\text { know how to handle. }\end{array}$ & $3.70(1.06)$ & $3.09(0.90)$ \\
\hline Note. $N_{\text {experimental group }}=23, N_{\text {control group }}=23$. & & \\
\end{tabular}

The analysis of covariance (ANCOVA) was conducted to test whether the MM-based training enhanced MM quality, pre-test measure of MM quality being the covariate. Results showed that neither the main effect of training $(F(1,45)=$ $0.85, n s)$ nor training-by-covariate interaction $(F(1,45)=$ $0.49, n s)$ was significant. This meant the MM-based training failed to enhance MM quality, thus provided no support for hypothesis 3. Means and standard deviations concerning MM quality across two conditions are reported in Table II.

TABLE II. MEANS, STANDARD DEVIATIONS OF MENTAL MODEL QUALITY IN EXPERIMENTAL GROUP AND CONTROL GROUP

\begin{tabular}{ccccc}
\hline & \multicolumn{2}{c}{ Pre-test } & \multicolumn{2}{c}{ Post-test } \\
\cline { 2 - 5 } & $M$ & $S D$ & $M$ & $S D$ \\
\hline Experimental group & 0.366 & 0.249 & 0.048 & 0.073 \\
Control group & 0.392 & 0.192 & 0.043 & 0.093 \\
\hline Note. $N_{\text {experimental group }}=23, N_{\text {control group }}=23$. & & &
\end{tabular}

\section{DISCUSSION}

This study developed a new training strategy i.e., MMbased training, and sought to examine whether it increased mental model quality or improved other indices of effective functioning. Despite the fact that its effect on MM quality was not observed in the current study, our newly-developed 
MM-based training strategy went beyond the cross-training approach to some extent. Findings suggested that MM-based training had a positive impact upon operators' reflective and learning behaviors, which has been proved to be helpful for individual or team performance. Given that the training was lasting only for two hours, the MM-based training strategy was promising in providing an efficient and feasible alternative for training complex system operators.

There were probably several reasons why MM-based training did not enhance post-test MM quality. The most salient reason might be that the time interval between training intervention and post-test was not enough for operators to foster MMs of higher quality [10]. Future study is needed to observe the effect of training on MM quality in a longer period. Moreover, operators were exposed to the new training strategy once. Although such training did result in a positive attitude and change of behaviors, it might be insufficient to improve operators' knowledge structure. In addition, the floor effect might exist in the post-test of MM. As indicated in Table I, the MM quality of post-test was far lower than pre-test. Future empirical study should be cautious of the equivalence of MM eliciting materials.

Results from this research have a number of practical implications. For individuals, the measurement of the taskrelated MM alone can be used as an effective self-reflection tool. It affords a way to explicitly depict one's knowledge structure of the task, which not only helps individuals to diagnose their weakness in knowledge structure but also deepen their understanding of the task. For teams, the interactive process between team members, which is highlighted in the MM-based training, can serve as a good way for team building. Effective interaction helps teammates to develop share mental models that facilitate coordination and reduce friction among team members. Moreover, it has some implications for designing training programs. First, the focus of training programs should be shifted to changing one's cognitive structure rather than teaching specific knowledge or skills, so that trainees are able to generalize what they have learned to novel situations with which they have not yet encountered. Second, a well-designed training program should try to develop trainees' perspective taking ability, which helps them find the access to see the whole picture and thus have a full understanding of key elements concerning the tasks and the interpersonal interactions. Last but not least, materials for training purpose should be highly relevant to real work situations to maximize the effectiveness of training transfer.

A few limitations should be highlighted. Firstly, our quasi-experimental design couldn't rule out the possibility that the differences between training and control groups were derived from other factors rather than the training intervention. Random assignment of participants is needed in future study. Secondly, we observed the effectiveness of training in a short time. A longer period is necessary so that training effects unfold fully and permeate the actual work process. Thirdly, we just implemented a single training session. Training effectiveness would be more salience when trainees are exposed to a series of training interventions.
In conclusion, we introduced a new training strategy, named mental model-based training, and provided some preliminary evidence of its effectiveness and advantages in a complex system situation. Further research is encouraged to optimize the training strategy, to provide more empirical evidence of its effectiveness, and to apply into practice.

\section{ACKNOWLEDGEMENT}

This study was supported by the National Natural Science Foundation of China (No. 71271203) and the Knowledge Innovation Program of CAS (KSCX2-EW-J-8). Correspondence should be sent to Xinwen Bai (baixw@psych.ac.cn).

\section{REFERENCES}

[1] P. N. Johnson-Laird, "Mental models and human reasoning," Proceedings of the National Academy of Sciences of the United States of America, vol. 107, pp. 18243-18250, 2010.

[2] W. B. Rouse, J. A. Cannon-Bowers, and E. Salas, "The role of mental models in team performance in complex systems," IEEE Transactions on Systems, Man, and Cybernetics, vol. 22, pp. 1296-1308, 1992.

[3] N. J. Cooke, P. A. Kiekel, E. Salas, R. J. Stout, C. A. Bowers, and J. A. Cannon-Bowers, "Measuring Team Knowledge: A Window to the Cognitive Underpinnings of Team Performance," Group Dynamics: Theory, Research, and Practice, vol. 7, pp. 179-199, 2003.

[4] M. D. Mumford, K. S. Hester, I. C. Robledo, D. R. Peterson, E. A. Day, D. F. Hougen, et al., "Mental Models and Creative ProblemSolving: The Relationship of Objective and Subjective Model Attributes," Creativity Research Journal, vol. 24, pp. 311-330, 2012.

[5] K. A. Smith-Jentsch, G. E. Campbell, D. M. Milanovich, and A. M. Reynolds, "Measuring teamwork mental models to support training needs assessment, development, and evaluation: Two empirical studies," Journal of Organizational Behavior, vol. 22, pp. 179-194, 2001

[6] B. D. Edwards, E. A. Day, W. Arthur, and S. T. Bell, "Relationships among team ability composition, team mental models, and team performance," Journal of Applied Psychology, vol. 91, pp. 727-736, 2006.

[7] A. P. J. Ellis, "System breakdown: The role of mental models and transactive memory in the relationship between acute stress and team performance," Academy of Management Journal, vol. 49, pp. 576589, 2006.

[8] M. A. Marks, S. J. Zaccaro, and J. E. Mathieu, "Performance implications of leader briefings and team-interaction training for team adaptation to novel environments," Journal of Applied Psychology, vol. 85, pp. 971-986, 2000.

[9] L. Van Boven and L. Thompson, "A look into the mind of the negotiator: Mental models in negotiation," Group Processes \& Intergroup Relations, vol. 6, pp. 387-404, 2003.

[10] K. A. Smith-Jentsch, J. A. Cannon-Bowers, S. I. Tannenbaum, and E. Salas, "Guided team self-correction - Impacts on team mental models, processes, and effectiveness," Small Group Research, vol. 39, pp. 303-327, 2008

[11] M. A. Marks, M. J. Sabella, C. S. Burke, and S. J. Zaccaro, "The impact of cross-training on team effectiveness," Journal of Applied Psychology, vol. 87, pp. 3-13, 2002.

[12] D. Kirkpatrick, "Techniques for evaluating training programs," Training \& Development, vol. 50, pp. 54-59, 1996.

[13] W. Arthur, W. Bennett, P. S. Edens, and S. T. Bell, "Effectiveness of training in organizations: A meta-analysis of design and evaluation features," Journal of Applied Psychology, vol. 88, pp. 234-245, 2003.

[14] A. Edmondson, "Psychological safety and learning behavior in work teams," Administrative Science Quarterly, vol. 44, pp. 350-383, 1999 